\title{
Multiphoton Microscopy in the Study of Morphological Characteristics of Radiation-Induced Injuries of the Bladder
}

DOI: 10.17691/stm2016.8.2.04

Received October 16, 2015

S.S. Kuznetsov, MD, DSc, Professor, Department of Pathological Anatomy';

V.V. Dudenkova, Junior Researcher, Institute of Biomedical Technologies'; PhD Student,

Department of General Physics²

M.V. Kochueva, PhD Student, Oncology, Radiotherapy, Radiodiagnosis Department';

E.B. Kiseleva, PhD, Junior Researcher, Institute of Biomedical Technologies';

N.Yu. Ignatieva, DSc, Associate Professor, Department of Physical Chemistry3;

O.L. Zakharkina, Researcher, Laser Chemistry Laboratory4;

E.A. Sergeeva, PhD, Senior Researcher, Department of Radio-Physical Methods in Hydrophysics ${ }^{5}$;

K.V. Babak, PhD Student, Department of Biophysics;

A.V. Maslennikova, MD, DSc, Professor, Oncology, Radiotherapy, Radiodiagnosis Department'; Professor,

Biophysics Department ${ }^{2}$

${ }^{1}$ Nizhny Novgorod State Medical Academy, 10/1 Minin and Pozharsky Square, Nizhny Novgorod, 603005,

Russian Federation;

'Lobachevsky State University of Nizhni Novgorod, 23 Prospekt Gagarina, Nizhny Novgorod, 603950,

Russian Federation;

'Lomonosov Moscow State University, 1, Bldg. 3 Leninskiye Gory, Moscow, 119991, Russian Federation;

${ }^{4}$ Institute on Laser and Information Technologies, Russian Academy of Sciences, 2 Pionerskaya St., Moscow,

Troitsk, 142092, Russian Federation;

${ }^{5}$ Institute of Applied Physics, Russian Academy of Sciences, 46 Ulianova St., Nizhny Novgorod, 603950,

Russian Federation

The aim of the investigation was to assess the feasibility of multiphoton microscopy (MPM) for studying dynamics of bladder structural changes following a single exposure to gamma-radiation at various doses (2, 10, and $40 \mathrm{~Gy})$ in experiment.

Materials and Methods. Specimens of rat bladders after a single local radiation at the dose of 2, 10, and 40 Gy were the objects of investigation (9 groups with two rats for each dose and term, and two intact rats -20 observations in all). The study was carried out 1 day, 1 week, and 1 month after radiation exposure. Part of the histological bladder preparations was stained with picrofuchsin according to Van Gieson method. The other part of the sections, obtained from the same blocks, was investigated using MPM without additional staining. For this purpose a laser scanning microscope LSM Axiovert 510 Meta (Carl Zeiss, Germany) was used. Excitation was generated with a femtosecond Ti:Sapphire laser (MAI TAI HP, Spectra Physics, USA) at the wavelength of $800 \mathrm{~nm}$, registration was performed in the range of $362-415 \mathrm{~nm}$ (second harmonic signal from collagen) and 512-576 nm (signal of two-photon excited elastin autofluorescence).

Results. Application of MPM method allowed us to find out, that in early terms (1 day and 1 week) after radiation exposure the process of alteration of collagen-containing structures of bladder walls was a leading one at all selected doses. A month after 2 and $10 \mathrm{~Gy}$ radiation increase in collagen structures was registered, speaking of the onset of radiation fibrosis formation. At a dose of $40 \mathrm{~Gy}$ decrease of second harmonic signal retained in the extracellular matrix of the bladder wall. It allowed us to draw a conclusion on a long-term disorganization of collagen at high radiation doses.

Conclusion. MPM method makes it possible to estimate, that structural destruction of extracellular tissue matrix occurs even after low radiation doses and in early terms after radiation exposure, which is not possible to reveal using standard microscopy. Duration of

For contacts: Kseniya V. Babak, e-mail: kseniya9127@mail.ru 
disorganization process of collagen-containing structures depends on the radiation dose: high doses result in longer-lasting alterations. MPM enables also the assessment of the course of restorative processes.

Key words: bladder injury; ionizing radiation; multiphoton microscopy; extracellular matrix; collagen.

Alterations of normal tissues, occurring in various terms after radiation therapy for malignant neoplasms, present one of the unsolved problems of modern radiation oncology [1]. Of special importance for organs of small pelvis (bladder and rectum) are radiation side effects, when they appear in patients with predicted long survival lifetime, who consider maintaining quality of life to be no less important than recovery from oncological disease [2].

A key moment in the pathogenesis of radiationinduced complications is generation of fibrous changes in the exposed zone due to excessive synthesis of collagen and replacement of the damaged cells and fibrillar structures by it [3]. During recent years main molecular and genetic mechanisms responsible for early and late radiation alterations of normal tissues have been studied and identified [3-5]. Investigation of radiation-induced alterations of collagen on a modeled medium allowed the researchers to find out the main molecular and tissue mechanisms of degradation and successive regeneration of it in different terms after a single exposure at increasing doses [6]. However, dynamics of collagen condition at various levels of its hierarchic organization (molecules, fibrils, fibers, bundles) remains unstudied during these processes. It should be underlined, that not only collagen but elastin as well is involved in the structural organization of fibrillar elements of internal organs. Detailed investigations of dose-time relations of the condition of collagen- and elastin-containing elements of internal organs, exposed to ionizing radiation, have not been carried out so far.

In recent years multiphoton laser scanning microscopy (MPM) is being actively implemented in morphological investigations. This method enables researchers to obtain structural images of biological tissues visualizing such components as nicotinamide adenine dinucleotide, flavoproteins, keratin, lipofuscin, elastin, melanin and porphirins by their autofluorescence due to two-photon excitation phenomenon $[7,8]$. Collagen is visualized due to second harmonic generation from the molecules having no center of symmetry [9, 10, 11]. Under the impact of a short-pulsed ( $100 \mathrm{fs}$ ) laser radiation with a high peak power collagen generates nonlinear response at a doubled frequency $[12,13]$. Scanning with a sharply focused laser beam makes it possible to obtain images reflecting specific arrangement of collagen in the tissue in detail. At present, application of two-photon excitation and second harmonic generation phenomena helps to solve a great number of experimental and clinical tasks ex vivo $[14,15]$ and in vivo $[15,16]$ in ophthalmology
$[17,18]$, dermatology [19, 20], cardiology [21], and also in experimental and clinical oncology [22-24].

The aim of the investigation was to assess the feasibility of multiphoton microscopy for studying dynamics of bladder structural changes following a single exposure to gamma-radiation at various doses $(2,10$, and $40 \mathrm{~Gy})$ in experiment.

Materials and Methods. Experiments were carried out on white outbred rats $(n=20)$, kept in standard conditions of vivarium. Animals were divided into 9 groups (two rats for each dose and term, and two unexposed rats). The work was performed in accordance with ethical principles established by European Convention for the Protection of Vertebrata used for Experimental and other Scientific Purposes (the Convention was passed in Strasburg, March, 18, 1986, adopted in Strasburg, June, 15, 2006) and approved by Bioethics Committee of Nizhny Novgorod State Medical Academy.

Radiation of the bladder was performed a single time by a local field at doses of 2, 10, and 40 Gy under general anesthesia (Zoletil $50 \mathrm{mg} / \mathrm{kg}$ ) using Terabalt unit for distant radiation therapy (UJP, Czech Republic) (beam energy - $1.25 \mathrm{MeV}$ ). Animals were withdrawn from the experiment in 1 day, 1 week, and 1 month using ether narcosis. The bladder was prepared, specimens for histological examination and MPM were fixed in $10 \%$ neutral formaldehyde solution for $24 \mathrm{~h}$. Then specimens were embedded in paraffin (Histomix extra medium, BioVitrum, Russia) with the following mounting of the paraffin blocks. Blocks were cut to 0.1 and $10 \mu \mathrm{m}$ sections on rotary microtome Leica $450 \mathrm{RM}$ (Leica Microsystems, Germany). Sections $0.1 \mu \mathrm{m}$ thick were stained by picrofuchsin according to Van Gieson. Specimens $10 \mu \mathrm{m}$ thick, obtained from the same blocks, were dewaxed and examined by MPM without additional staining. Bladder preparations of unexposed animals were used as controls.

Investigations were performed on inverted laser scanning microscope (LSM Axiovert 510 Meta, Carl Zeiss, Germany). A short-pulsed femtosecond laser (MAI TAI HP, Spectra Physics, USA) with a repetition rate of $80 \mathrm{MHz}$ and duration of $100 \mathrm{fs}$ was used as a source of exciting radiation. Images of tiny structure were obtained with the help of $40 \times / 1.3$ oil-emersion objective (EC Plan Neofluar, Carl Zeiss, Germany), which allowed us to achieve $318 \times 318 \mu \mathrm{m}$ field of vision with resolution of $1,024 \times 1,024$ pixels.

The following spectral characteristics of the visualization system were used: excitation at $800 \mathrm{~nm}$ 
wavelength, registration in two wavelength spectral ranges: $362-415 \mathrm{~nm}$, which had a peak of nonlinear response at second harmonic (400 nm) [12], and 512-576 nm, which corresponded to the range of autofluorescence of elastin under two-photon excitation [21]. Registration was performed in the mode of fully opened pinhole.

Results. At the initial stage of the work MPM investigation of topographic characteristics of collagenand elastin-containing structures of the bladder unexposed to radiation was carried out. On MPM images a mucous membrane with its proper plate, submucosal base and elements of the wall muscular layer (Figure 1 (a), white arrow) are well discerned. Epithelium of the bladder mucosa (urothelium) was presented by an even sheet of closely adjoining cells, connective tissue elements in the intercellular spaces not being found (Figure 1 (a), red arrow). Basement membrane is arranged as a combination of collagen and elastic fibers, the latter prevailing, and features by a regular contour, repeating the motion of the epithelial sheet (Figure 1 (a), yellow arrow). It is $21 \mu \mathrm{m}$ thick. In the proper mucous plate and submucosal layer, presented by a network of collagen fibers with a small admixture of elastic and some quantity of muscular elements, arterioles, venules and capillaries containing only collagen fiber endothelium in the vascular adventitias and endothelial basement membrane, were determined. Muscular fibers formed bundles of about the same thickness, going in various directions according to their functions. Between the muscular bundles there was a small number of collagen fibers with single elastic fibers, which presented a framework of the muscular layer (Figure 1 (a), blue arrow). The collagen- and elastin-containing elements in the rat intact bladder were mainly located in: urothelial basement membrane, proper mucous plate, submucosal layer, blood vessel walls and stromal framework of the muscular coat. Collagen/elastic fibers ratio was different in various structures. Elastic fibers prevailed in the epithelial basement membrane; proper mucous plate, submucosal layer, blood vessel walls and stromal framework of the muscular coat demonstrated predominant content of collagen fibers.

Alterations found in the bladder wall 1 day after irradiation, depended significantly on the amount of radiation load. After the exposure at a dose of $2 \mathrm{~Gy}$, slight reduction in contour sharpness of the urothelial basement membrane has been noted on MPM images, and a slight swelling of the main substance of extracellular matrix, located between the collagen- and elastin-containing elements of the submucosal layer, though considerable change of the basement membrane thickness and the ratio of collagen-/elastin-containing structures were not observed (Figure 2 (a), upper row). Alteration data may indicate to the superficial disorganization of extracellular matrix (mucoid swelling). No disturbances of connective tissue elements of the blood vessel walls and muscle layer were found. It should be noted, that the above described alterations of the bladder a day after 2 Gy radiation are not determined by a routine light microscopy (Figure 2 (a), lower row).

One day after radiation at a dose of 10 Gy edema of the submucosal layer was determined on MPM images, appearing in the form of fibril thickening and increase of the distance between the structural elements of extracellular matrix (Figure 2 (b), upper row). In the urothelial basement membrane there was noted fragmentation of collagen fibers and disruption of interrelations of elastin- and collagen-containing structures, i.e. destruction of connective tissue structures
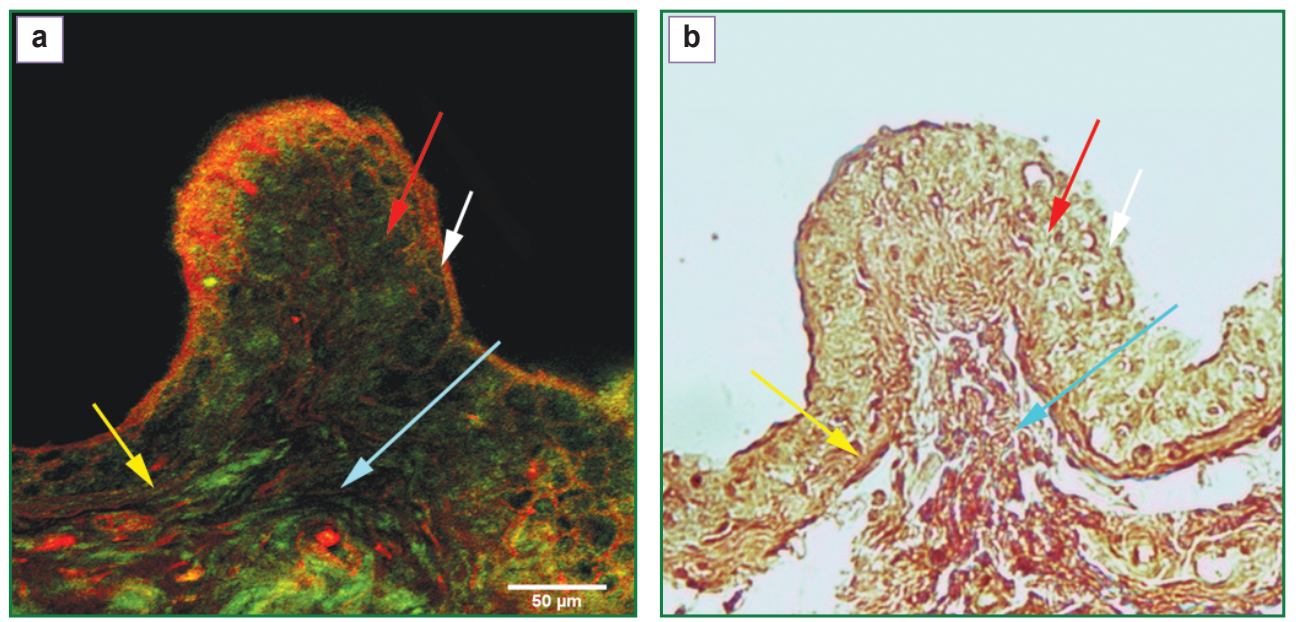

Figure 1. Rat intact bladder: (a) MPM image (here and further on other figures: image superposition of second harmonic and two-photon autofluorescence signals); (b) image of histological preparation stained by picrofuchsin according to Van Gieson, x40; oil immersion was used to obtain MPM images. See the description in the text 

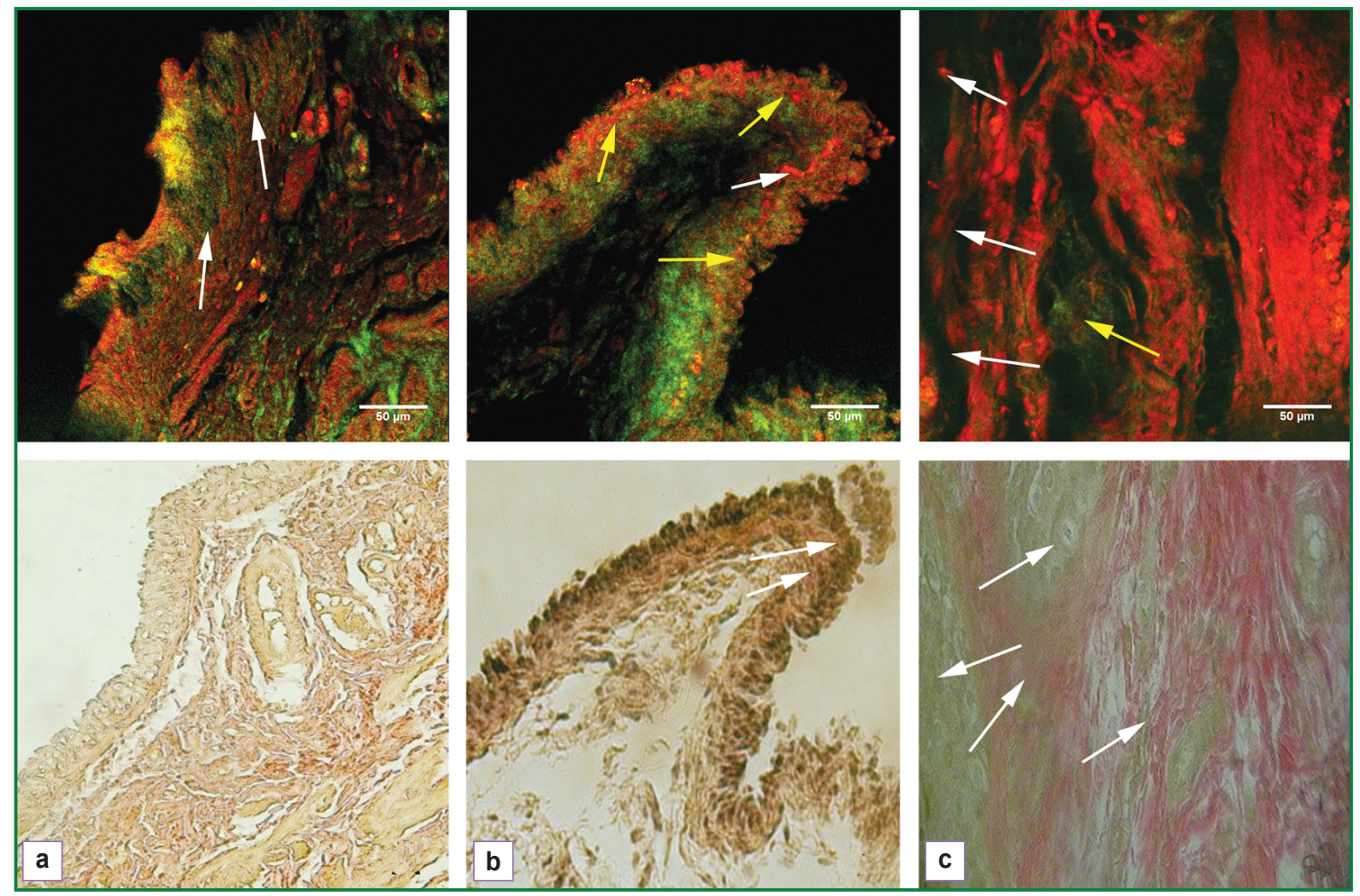

Figure 2. Morphological alterations in the rat bladder 1 day after radiation exposure (the upper row represents MPM images, the lower row shows images of histological specimens stained according to Van Gieson): (a) after radiation at a dose of 2 Gy: decreased definition of urothelial basement membrane contours and a slight separation of connective tissue and elastic elements of submucosal layer without changes in basement membrane thickness and collagen-/elastin-containing structure ratio (white arrows), seen on MPM images; (b) after radiation at a dose of $10 \mathrm{~Gy}$ : fibril thickening and increase of the distance between the structural elements of extracellular matrix speak of the presence of edema (white arrows), fragmentation of collagen fibers and violation of interrelations of elastin- and collagen-containing structures in the urothelial basement membrane (yellow arrows); (c) after radiation at a dose of $40 \mathrm{~Gy}$ : availability of edema in the proper mucous plate and submucosal base, concurrently a focal destruction of the collagen-containing structures involving about half the connectivetissue elements (white arrows) are noted in the submucosal layer, in the proper mucous plate small elements are seen which give a weak signal of second harmonic generation from an insignificant amount of remained collagen-containing structures

took place, the process not visualized by conventional microscopy (Figure 2 (b), lower row).

One day after 40 Gy radiation exposure expected more serious destructive processes were found in the fibrous framework on MPM images. Edema was seen in the proper mucous plate and submucosal base, while focal destruction of collagen-containing structures (Figure 2 (c), yellow arrow), involving about half the connective tissue elements was also revealed in sbmucosal layer. Almost complete absence of the signal in the range of 352-416 $\mathrm{nm}$ (See Figure 2 (c), upper row) was notable. In the proper mucous plate small elements were determined, giving a signal to generate second harmonic, which seemed to be incompletely destructed collagen-containing objects.

Dynamic observation of the fibrillar structure condition was continued 1 week after the radiation exposure. After 2 Gy radiation irregularity, discontinuity and attenuation of the signal in the range of $362-416 \mathrm{~nm}$ were determined in the zone of urothelium, its basement membrane and submucosal layer on MPM images (Figure 3 (a), upper row). All these changes reflected alterations, which structural manifestations were blurring of the epithelial basement membrane contours, breaks in it, and, in general, the whole picture was similar to that usually observed in histological specimens (Figure 3 (b), lower row). Within the urothelial basement membrane single tiny collagen fibers were found differing from the previously observed fibers in the intensity of MPM signal, which was estimated by us as neocollagenogenesis (the beginning of reparative processes) (See Figure 3 (a), upper row). No changes in the fibrous elements of the blood vessels and the framework of the muscular layer were observed.

One week after radiation exposure at $10 \mathrm{~Gy}$ alteration processes were found in all departments of 

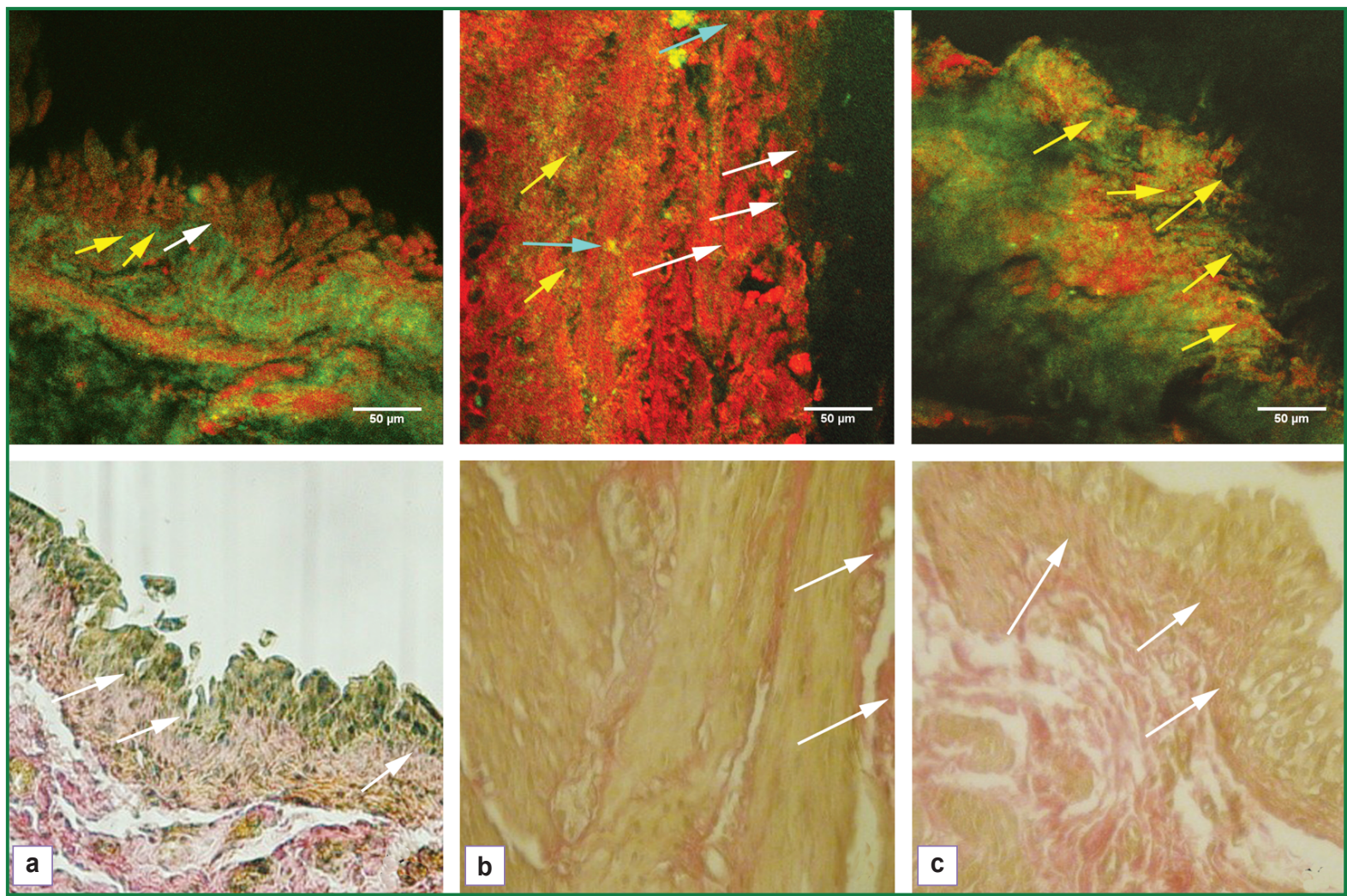

Figure 3. Morphological alterations in the rat bladder 1 week after radiation exposure (the upper row represents MPM images, the lower row shows images of histological specimens stained according to Van Gieson): (a) after radiation at a dose of 2 Gy: contour blurring and ruptures of the epithelial basement membrane (white arrows), new-formed thin collagen fibers within the basal membrane (yellow arrows); (b) after radiation at a dose of $10 \mathrm{~Gy}$ : absence of epithelium, its desquamation, fragmentation of the fibrous structures of the basement membrane and submucosal layer (white arrows), destruction of muscular fibers (yellow arrows), elements of the new-formed collagen fibers (blue arrows); (c) after radiation at a dose of 40 Gy: gross deformation of collagen fibers with the decrease of their quantity (white arrows), on MPM images the range of fibrous structure destruction is more significant: areas with impaired optical properties of epithelium and its basement membrane, fibrous structures of the submucosal layer, reflecting their damage, not visualized by conventional microscopy (yellow arrows)

the bladder wall in the form of large areas of urothelium desquamation, fragmentation of the fibrous structures of the basement membrane and submucosal layer. Destruction of the collagen fibers in the form of their homogenization and fragmentation was brighter on MPM images compared to standard staining, which was reflected as diffused reduction of the signal in the range of 362-416 nm (Figure 3 (b), upper row). Among the elements of the destructed collagen thin collagencontaining fibrils without signs of disorganization were visualized, which were referred to newly-formed fibers. At the same time, destruction of smooth muscle fibers was defined, which conventional histological examination failed to reveal (See Figure 3 (b), lower row).

The picture of structural changes, found 1 week after laser exposure at a dose of 40 Gy by ordinary microscopy did not differ much from the changes noted 1 day after the exposure at the same dose, and was characterized by a gross deformation of collagen fiber bundles and reduction of their quantities (Figure 3 (c), lower row). The range of fibrous structure damage on MPM images was significant: a general anatomic structure of the mucous membrane was broken, the epithelium desquamated, in some areas complete destruction of the urothelial basement membrane was clearly seen (second harmonic generation signal in the range of $362-416 \mathrm{~nm}$ was almost fully absent). Edema with chaotic arrangement of fibers and their fragmentation was observed in the muscular layer. But there were no signs of new collagen- and elastincontaining structures (Figure 3 (c), upper row). A "delay" of the reparative processes was likely to occur caused by blockage of their triggering due to a high irradiating dose used.

Investigations of the bladder specimens 1 month after irradiation at the dose of 2 Gy showed no signs of 


\section{BIOMEDICAL INVESTIGATIONS}

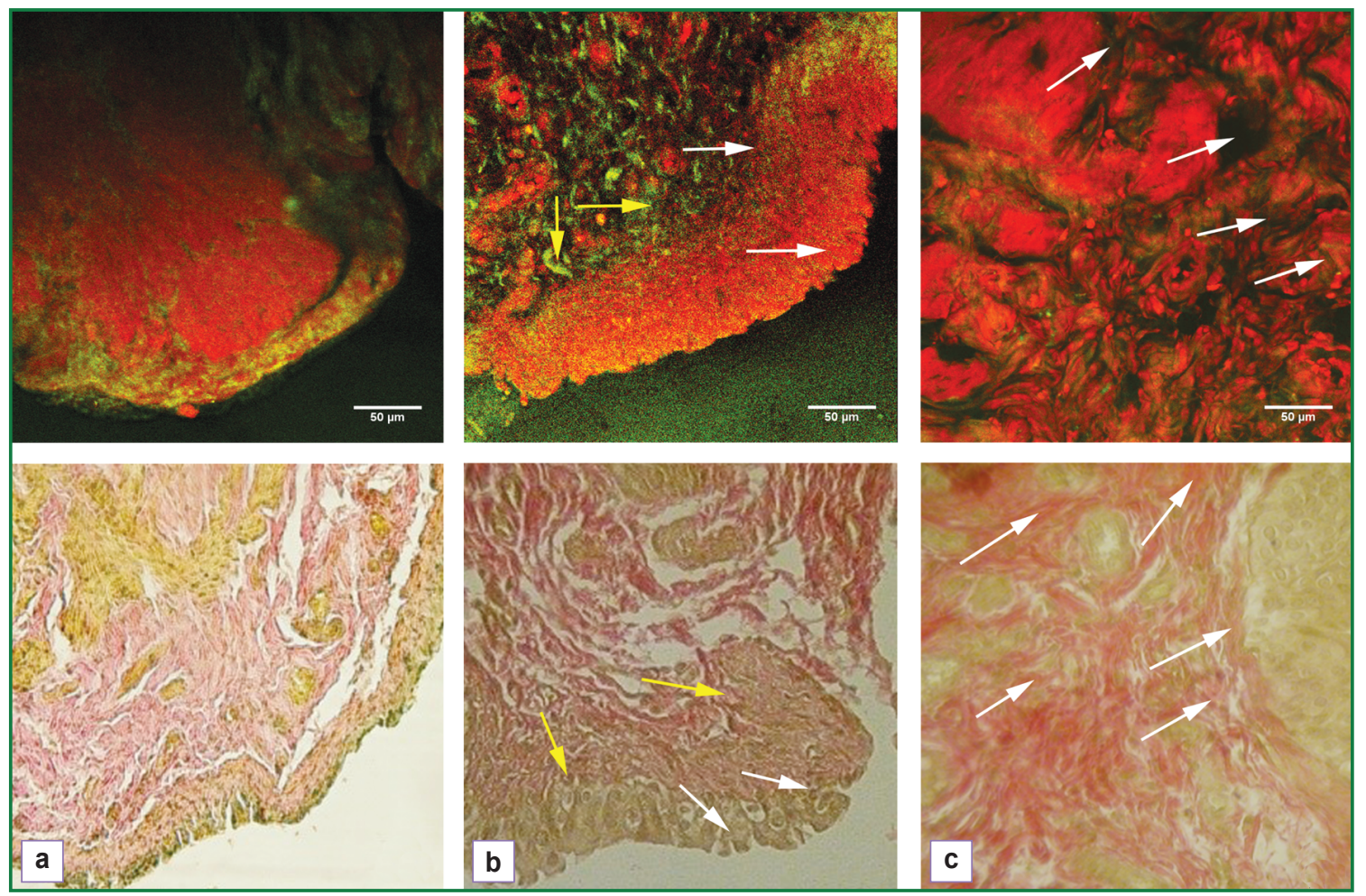

Figure 4. Morphological alterations in the rat bladder 1 month after radiation exposure (the upper row represents MPM images, the lower row shows images of histological specimens stained according to Van Gieson): (a) after radiation at a dose of 2 Gy: no evidence of alteration of collagen-, elastin-containing elements is seen either on MPM images or on the preparations stained according to Van Gieson; (b) after radiation at a dose of 10 Gy: "loosening" of the urothelial sheet (white arrows), signs of vessel cell sclerosis in the submucosal layer with narrowing and obliteration of the lumens of some vessels of the connective tissue, fibrous alterations (yellow arrows); (c) after radiation at a dose of 40 Gy: diffuse sclerosis of the urothelial basement membrane, submucosal base and muscular layer with the appearance of rough fibrous connective tissue with extensive hyalinosis of the collagen fibers, which does not give typical signal on MPM images (white arrows)

alteration of collagen- and elastin-containing elements both on MPM images (Figure 4 (a), upper row) and on the preparations stained according to Van Gieson (Figure 4 (a), lower row).

Morphological changes found 1 month after exposure at a dose of $10 \mathrm{~Gy}$ were characterized by "loosening" of the urothelial sheet, which was likely due to weakening of the intercellular interconnections caused by a high content of the destroyed connective tissue elements in the basement membrane, muscular plate of the mucous membrane and submucosal layer. In the submucosal layer, in its turn, signs of the generating fibrosis of the extracellular matrix and reduction of the vessel bed were detected (development of vessel wall sclerosis with narrowing and obliteration of lumens of some connective tissue vessels).

Studying the bladder 1 month after application of 40 Gy dose, diffuse sclerosis of the urothelial basement membrane, submucosal base and muscular layer with the rough fibrous connective tissue with extensive hyalinosis of the collagen fibers was revealed in the preparations stained according to Van Gieson (Figure 4 (c), lower row). This type of collagen did not give second harmonic generation signal on MPM images (Figure 4 (c), upper row).

Discussion. In this study investigation of morphological bladder wall changes in experimental animals in various terms after a single local gammairradiation at doses of 2, 10, and 40 Gy was performed by MPM method. The main biomedical applications of this method have been connected so far with the diagnosis of normal and pathological tissues [25]. In our study this method was used for the first time for dynamic state assessment of the organ exposed to ionizing radiation.

Two parallel processes: destruction (disorganization) and restoration of the damaged structures of collagencontaining elements have been found to occur in the bladder by histological examination and application of MPM method. Disorganization of the fibrous framework of the bladder wall structural elements was displayed in the form of swelling, fibrinoid changes and fragmentation of collagen elements and even their complete destruction at high radiation doses (40 Gy) 
under histological examination carried out according to Van Gieson method. Diffuse attenuation of the signal in the range of $362-416 \mathrm{~nm}$ corresponded to these alterations on MPM images. Similar changes (intensity reduction of second harmonic generation signal of the collagen) were detected during in vivo monitoring of the skin condition after thermal burning [26], and also in systemic diseases characterized by disorganization of the connective tissue structures of the inner organs [27]. Our investigation has confirmed, that this phenomenon can be considered a universal sign of disorganization of connective tissue matrix structure. MPM method enabled us to observe the process of fibrous structure damaging in the earlier terms and at less radiation doses, than in routine histological examination (1 day after the exposure at a dose of $2 \mathrm{~Gy}$ ). It has been demonstrated, that the processes of damage development and subsequent remodeling of extracellular organ matrix exposed to ionizing radiation occur in a stage-by-stage manner and start with disorganization and degradation of collagen at the level of fibers and bundles, which can be found even in the early terms after the exposure at 2 Gy dose. The next stage of tissue response to the radiation damaging effect is restoration - the process of neocollagenogenesis, which has been registered to start already 1 week after the detrimental exposure. Besides, the rate of the extracellular matrix remodeling processes has been estimated to depend on the radiation dose and to decrease essentially at higher radiation doses (40 Gy). The investigation conducted opens new possibilities of studying the condition of normal tissues exposed to ionizing irradiation. Further work in this direction should be connected with the developing methods of monitoring radiation-induced damages in vivo with the help of MPM method.

Conclusion. Method of multiphoton microscopy, in contrast to traditional histological examination, allows the detection of degradation of the bladder tissue collagen and its restoration in earlier terms and at a less radiation dose, making it possible to assess and correct the dynamics of radiation alterations in extracellular matrix.

Study Funding. The work was supported by the Russian Scientific Fund (project No.14-25-00055, investigations of radiation-induced alterations performed by laser scanning microscopy), Russian Fundamental Research Fund (project No.16-02-00670, performance of radiation exposure, investigation of radiation-induced alterations using standard histological examination method).

Conflicts of Interest. The authors declare no conflicts of interest related to this study.

\section{References}

1. Dörr W. Radiation effect in normal tissue - principles of damage and protection. Nuklearmedizin 2010; 49(Suppl 1): S53-S58.
2. Fiorino C., Valdagni R., Rancati T., Sanguineti G. Dosevolume effects for normal tissues in external radiotherapy: pelvis. Radiother Oncol 2009; 93(2): 153-167, http://dx.doi. org/10.1016/j.radonc.2009.08.004.

3. Yarnold J., Brotons M.C.V. Pathogenetic mechanisms in radiation fibrosis. Radiother Oncol 2010; 97(1): 149-161, http://dx.doi.org/10.1016/j.radonc.2010.09.002.

4. Stewart F.A., Akleyev A.V., Hauer-Jensen M., Hendry J.H., Kleiman N.J., Macvittie T.J., Aleman B.M., Edgar A.B., Mabuchi K., Muirhead C.R., Shore R.E., Wallace W.H. ICRP publication 118: ICRP statement on tissue reactions and early and late effects of radiation in normal tissues and organs - threshold doses for tissue reactions in a radiation protection context. Ann ICRP 2012; 41(1-2): 1-322, http://dx.doi.org/10.1016/j.icrp.2012.02.001.

5. Brush J., Lipnick S.L., Phillips T., Sitko J., McDonald J.T., McBride W.H. Molecular mechanisms of late normal tissue injury. Semin Radiat Oncol 2007; 17(2): 121130, http://dx.doi.org/10.1016/j.semradonc.2006.11.008.

6. Maslennikova A., Kochueva M., Ignatieva N., Vitkin A., Zakharkina O., Kamensky V., Sergeeva E., Kiseleva E., Bagratashvili V. Effects of gamma irradiation on collagen damage and remodeling. Int J Radiat Biol 2015; 91(3): 240 247, http://dx.doi.org/10.3109/09553002.2014.969848.

7. Göppert-Mayer M. Über elementarakte mit zwei quantensprüngen. Ann Phys 1931; 401(3): 273-295, http:// dx.doi.org/10.1002/andp.19314010303.

8. Alex A., Weingast J., Weinigel M., Kellner-Höfer M., Nemecek R., Binder M., Pehamberger H., König K., Drexler W. Three-dimensional multiphoton/optical coherence tomography for diagnostic applications in dermatology. J Biophotonics 2013; 6(4): 352-362, http://dx.doi.org/10.1002/ jbio. 201200085.

9. Denk W., Strickler J.H., Webb W.W. Two-photon laser scanning fluorescence microscopy. Science 1990; 248(4951): 73-76, http://dx.doi.org/10.1126/science.2321027.

10. Theodossiou T.A., Thrasivoulou C., Ekwobi C., Becker D.L. Second harmonic generation confocal microscopy of collagen type I from rat tendon cryosections. Biophys J 2006; 91(12): 4665-4677, http://dx.doi.org/10.1529/ biophysj.106.093740.

11. Seidenari S., Arginelli F., Bassoli S., Cautela J., French P.M., Guanti M., Guardoli D., König K., Talbot C., Dunsby C. Multiphoton laser microscopy and fluorescence lifetime imaging for the evaluation of the skin. Dermatol Res Pract 2012; 2012: 810749, http://dx.doi. org/10.1155/2012/810749.

12. Williams R.M., Zipfel W.R., Webb W.W. Interpreting second-harmonic generation images of collagen I fibrils. Biophys J 2005; 88(2): 1377-1386, http://dx.doi.org/10.1529/ biophysj.104.047308.

13. Raub C.B., Unruh J., Suresh V., Krasieva T., Lindmo T., Gratton E., Tromberg B.J., George S.C. Image correlation spectroscopy of multiphoton images correlates with collagen mechanical properties. Biophys J 2008; 94(6): 2361-2373, http://dx.doi.org/10.1529/biophysj.107.120006.

14. Chen J., Wong S., Nathanson M.H., Jain D. Evaluation of Barrett esophagus by multiphoton microscopy. Arch Pathol Lab Med 2014; 138(2): 204-212, http://dx.doi.org/10.5858/ arpa.2012-0675-OA.

15. Dimitrow E., Ziemer M., Koehler M.J., Norgauer J., König K., Elsner P., Kaatz M. Sensitivity and specificity of multiphoton laser tomography for in vivo and ex vivo diagnosis 
of malignant melanoma. J Invest Dermatol 2009; 129(7): 1752-1758, http://dx.doi.org/10.1038/jid.2008.439.

16. Balu M., Kelly K.M., Zachary C.B., Harris R.M., Krasieva T.B., König K., Durkin A.J., Tromberg B.J. Distinguishing between benign and malignant melanocytic nevi by in vivo multiphoton microscopy. Cancer Res 2014; 74(10): 2688-2697, http://dx.doi.org/10.1158/0008-5472.CAN-132582.

17. Huang A.S., Gonzalez J.M. Jr., Le P.V., Heur M., Tan J.C. Sources of structural autofluorescence in the human trabecular meshwork. Invest Ophthalmol Vis Sci 2013; 54(7): 4813-4820, http://dx.doi.org/10.1167/iovs.12-11235.

18. Morishige N., Yamada N., Zhang X., Morita Y., Yamada N., Kimura K., Takahara A., Sonoda K.H. Abnormalities of stromal structure in the bullous keratopathy cornea identified by second harmonic generation imaging microscopy. Invest Ophthalmol Vis Sci 2012; 53(8): 49985003, http://dx.doi.org/10.1167/iovs.12-10214.

19. Koehler M.J., König K., Elsner P., Bückle R., Kaatz M. In vivo assessment of human skin aging by multiphoton laser scanning tomography. Opt Lett 2006; 31(19): 2879-2881, http://dx.doi.org/10.1364/ol.31.002879.

20. Tsai T.H., Jee S.H., Dong C.Y., Lin S.J. Multiphoton microscopy in dermatological imaging. J Dermatol Sci 2009; 56(1): 1-8, http://dx.doi.org/10.1016/j.jdermsci.2009.06.008.

21. Cicchi R., Matthäus C., Meyer T., Lattermann A., Dietzek B., Brehm B.R., Popp J., Pavone F.S. Non-linear imaging and characterization of atherosclerotic arterial tissue using combined two photon fluorescence, secondharmonic generation and CARS microscopy. Proc.
SPIE 8948, Multiphoton Microscopy in the Biomedical Sciences XIV, 894807 (February 28, 2014), http://dx.doi. org/10.1117/12.203701822.

22. Chen W.S., Wang Y., Liu N.R., Zhang J.X., Chen R. Multiphoton microscopic imaging of human normal and cancerous oesophagus tissue. J Microsc 2014; 253(1): 79-82, http://dx.doi.org/10.1111/jmi.12102.

23. Paoli J., Smedh M., Ericson M.B. Multiphoton laser scanning microscopy - a novel diagnostic method for superficial skin cancers. Semin Cutan Med Surg 2009; 28(3): 190-195, http://dx.doi.org/10.1016/j.sder.2009.06.007.

24. Burke K., Tang P., Brown E. Second harmonic generation reveals matrix alterations during breast tumor progression. J Biomed Opt 2012; 18(3): 031106, http://dx.doi. org/10.1117/1.JBO.18.3.031106.

25. Campagnola $P$. Second harmonic generation imaging microscopy: applications to diseases diagnostics. Anal Chem 2011; 83(9): 3224-3231, http://dx.doi.org/10.1021/ac1032325.

26. Yasui T., Tanaka R., Hase E., Fukushima Sh., Araki T. In vivo time-laps imaging of skin-burn wound healing using second-harmonic generation microscopy. Proc. SPIE 8948, Multiphoton Microscopy in the Biomedical Sciences XIV, 89480B (February 28, 2014), http://dx.doi. org/10.1117/12.2038022.

27. Kiseleva E., Kirillin M., Feldchtein F., Vitkin A., Sergeeva E., Zagaynova E., Streltzova O., Shakhov B., Gubarkova E., Gladkova N. Differential diagnosis of human bladder mucosa pathologies in vivo with cross-polarization optical coherence tomography. Biomed Opt Express 2015; 6(4): 1464-1476, http://dx.doi.org/10.1364/BOE.6.001464. 